\title{
Lernen aus Fehlern anhand eines fallbasierten Curriculums im medizinischen Querschnittsbereich Gesundheitssysteme] Gesundheitsökonomie und öffentliche Gesundheitspflege
}

Learning from Mistakes using a Case-based Curriculum in Medical Education for Health Systems/health Economics and Public Health Care

Autoren

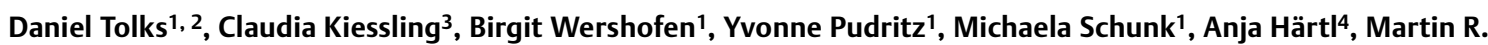
Fischer $^{1}$, Johanna Huber ${ }^{1}$

Institute

1 Institut für Didaktik und Ausbildungsforschung in der Medizin, Klinikum der Universität München, LMU München

2 Zentrum fur Angewandte Gesundheitswissenschaften, Leuphana Universität Lüneburg, Lüneburg

3 Lehrstuhl für die Ausbildung personaler und interpersonaler Kompetenzen im Gesundheitswesen, Universität Witten/Herdecke, Witten

4 Abteilung Medizindidaktik Augsburg, Medizinische Fakultät, Universität Augsburg, Augsburg

\section{Schlüsselwörter}

Deutsches Gesundheitssystem, Fallbasiertes Lernen, Game-Based Learning, Medizinische Ausbildung,

Globalisierung, Interprofessionell

Key words

Game-Based Learning, Case-Based Learning, Globalization, Medical Education, Interprofessional, German Health Care System

Korrespondenzadresse

Dr. Daniel Tolks

Institut für Didaktik und Ausbildungsforschung in der Medizin Klinikum der Universität München, LMU München

Pettenkoferstraße 8a

80336 München

daniel.tolks@med.uni-muenchen.de

\section{ZUSAMMENFASSUNG}

Ziel der Studie Es bestehen kaum Konzepte, wie den Studierenden Wissen über das eigene Gesundheitssystem vermittelt und die Wichtigkeit und Praxisrelevanz des Themas verdeutlicht werden kann. Der fallbasierte Ansatz und die darin beschriebenen Fehler sollen die Relevanz des Themas für die ärztliche Tätigkeit hervorheben.

Methodik Es wurde ein Kurskonzept entwickelt, welches die Praxisrelevanz der Inhalte den Studierenden möglichst nah gebracht werden kann. Dieses basiert auf einem Methodenmix aus Game-Based Learning, fallbasierten und kooperativen Lernens. Im Rahmen des Seminars wird der Weg eines krebskranken Patienten durch das Gesundheitswesen beschrieben, der aufgrund mangelnder Absprachen und weiterer Schnittstellenproblematiken unbefriedigend behandelt wird. Der fallbasierte Ansatz und die darin beschriebenen Fehler sollen die Relevanz des Themas für die ärztliche Tätigkeit hervorheben.

Ergebnisse Analysen zeigen, dass die Studierenden $(n=1162)$ bei beiden Erhebungszeiträumen ihr Interesse am Seminarthema eher im mittleren Bereich einordneten. Dennoch empfanden die Studierenden die Methode des fallbasierten Lernens als gut und schätzten die Relevanz des Themas als hoch ein. Schlussfolgerungen Die spätere Relevanz des Themenkomplexes GGG für die berufliche Tätigkeit wurde von den Studierenden scheinbar erkannt. Die geringe Motivation der Studierenden, sich mit der Thematik zu beschäftigen, konnte scheinbar reduziert werden, was auch durch die rückgemeldeten Beobachtungen der Dozierenden bestätigt wurde.

\section{ABSTRACT}

Aim of the study There are hardly any concepts on how to impart knowledge about the own health system to students and to clarify the importance and practical relevance of the topic. The case-based approach and the errors described 
therein should highlight the relevance of the topic to the medical profession.

Methodology A course concept was developed with focus on the practical relevance of the content to students. This was based on a method mix of game-based learning, case-based and cooperative learning. The seminar describes the path of a cancer patient through health care, an issue which, due to a lack of agreements and other interface problems, is dealt with unsatisfactorily.
Results Analyses showed that students $(n=1162)$ had moderate interest in the topic of the seminar during both survey periods. However, they found the method of case-based learning to be good and rated the relevance of the topic as high. Conclusions The relevance of the topic complex GGG for later professional activity was apparently recognized by the students. The low motivation of the students to engage with this topic could be reduced, as confirmed by reports of the lecturers.

\section{Hintergrund}

Die Globalisierung und wachsende finanzielle Restriktionen lassen die Anforderungen an Gesundheitssysteme international steigen. Für Studierende der Human- und Zahnmedizin sowie der Pflege-, Gesundheits- und Wirtschaftswissenschaften wird daher ein fundiertes Verständnis der Gesundheitssysteme immer relevanter [15]. In einer sich stetig verändernden Welt und sich wechselseitig beeinflussenden Rahmenbedingungen stehen Gesundheitssysteme vor komplexen Aufgabenstrukturen, die sich auf die Ausbildung der Gesundheitsexperten auswirken und in den unterschiedlichen Ausbildungssystemen abgebildet werden [1,6-11]. Die Ausbildung von Gesundheitsexperten kann sogar als entscheidend für die Stärkung der Gesundheitssysteme angesehen werden [9, 12]. Die heutigen Studierenden werden die zukünftigen Entscheidungsträger im Gesundheitssektor sein und stellen damit einen wichtigen Faktor für die erfolgreiche Bewältigung der neuen Herausforderungen in den Gesundheitssystemen dar [13].

An die Einrichtungen und Beschäftigten des Gesundheitssystems wird zunehmend die Forderung gestellt, die gegenwärtige Fragmentierung und das häufig auftretende, isolierte Nebeneinander der verschiedenen Versorgungsinstitutionen zu überwinden, um die Versorgungsabläufe sektor-, institutions- und einrichtungsübergreifend organisieren zu können. Verbesserte Kooperation und Koordination im Gesundheitswesen erfordern v.a. Wissen über die Strukturen und Funktionsweise, nicht nur des eigenen Bereichs, sondern auch anderer Sektoren. Wer die Struktur- und Funktionslogik der anderen Teilsysteme kennt, kann die Handlungslogik seiner Interaktionspartner des anderen Teilsystems besser verstehen $[14,15]$.

In einer Studie von Behmann und Kollegen wurde die Durchführung der medizinischen Lehre im Querschnittsbereich (Q3) Gesundheitsökonomie, Gesundheitssystem, öffentliche Gesundheitspflege (GGG) erfasst [16]. Die Autoren stellten fest, dass zur Vermittlung dieser Themengebiete primär die Unterrichtsformen Seminare und Vorlesungen angeboten wurden. Die Kurse fanden in höheren Semestern (8.-10. Semester) statt. Die wichtigsten und am häufigsten unterrichteten Inhalte im Querschnittsbereich waren: „Die Finanzierung des Gesundheitssystems“, „Grundbegriffe und Zusammenhänge (Gesundheitsökonomie)“ sowie „Die Akteure im Gesundheitssystem“ (ebenda). Zwischen den 3 Thematiken des Querschnittsbereichs bestehen zwar inhaltliche Überschneidungen, allerdings wird die Lehre von unterschiedlichen Abteilungen oder Professionen durchgeführt. Zudem sind nicht an allen medizinischen Fakultäten Institute vorhanden, die die unterschiedlichen Themengebiete ver- treten können. Die Folge ist eine heterogene Umsetzung des Querschnittbereichs, was sich in unterschiedlichen Lerninhalten und variierendem Lehrumfang an den jeweiligen Fakultäten zeigt (ebenda). Behmann und Kollegen führten als weiteres Problem die Schwierigkeit an, dass die Verknüpfung der eher theoretisch orientierten Inhalte aus dem Querschnittsbereich mit den sonstigen medizinischklinischen Inhalten des Studiums zu einer niedrigeren Motivation bei den Studierenden führte, sich mit diesem Themenkomplex zu beschäftigen [16]. Weitere Studien bestätigen, dass die Studierenden nur über geringe Kenntnisse des eigenen Gesundheitssystems verfügen, obwohl ein Interesse an der Thematik besteht $[7,17,18]$.

Es ist daher notwendig, auf den veränderten Bedarf an Lehre im Bereich Gesundheitssystemvergleich zu reagieren und Lerner-zentrierte Curricula zu entwickeln um nicht zuletzt auch das Interesse an dem Themenbereich zu verbessern, bzw. überhaupt zu evozieren. Bislang existiert in Deutschland keine Studie zu innovativen Lehr-und Lernkonzepten im Bereich Gesundheitssysteme, Gesundheitsökonomie und öffentliches Gesundheitswesen, wie eine Datenbanksuche mit GoogleScholar und PubMed ergeben hat (Suchbegriffe: PubMed, GoogleScholar: „Lehrinnovation“, „medizinische Ausbildung“, „Gesundheitssysteme“, „Gesundheitsökonomie“, „öffentliche Gesundheitspflege“, „Studie“).

\section{Lernen aus Fehlern}

Laut Oser et al. kann davon ausgegangen werden, dass jeder Fehler Lernpotential beinhaltet [19]. Der Lernende muss den Fehler dabei gezielt als Lernanlass nutzen. Dazu muss der Fehler erkannt und die richtige Lösung nachvollzogen werden [20]. Erst der nachvollzogene Fehler, der zu Negativem Wissen geworden ist, befähigt den Lernenden, beim nächsten Mal Fehlerhaftes leichter zu erkennen. Zudem bleibt der Fehler im Gedächtnis und kann bei einem ähnlichem Problem angewandt werden [20]. Laut Oser muss der Fehler nicht selbst begangen worden sein, es ist ebenso möglich aus den Fehlern anderer Personen zu lernen (advokatorisches Lernen) [21].

\section{Situation an der Fakultät Medizin der LMU München}

In den Fächern Gesundheitssysteme/-ökonomie und öffentliche Gesundheitspflege (GGG) und Prävention und Gesundheitsförderung (P\&G) sah das Medizinische Curriculum München (MeCuM) der Ludwig Maximilians-Universität München (LMU) bis Sommersemester 2013 vor, dass die Studierenden eine Vorlesung besuchen und sowohl einem verpflichtendem Seminar als auch einer Exkursion zugeteilt wurden. Interne Rückmeldungen der Studierenden ergaben eine 
geringe Zufriedenheit mit der thematischen Ausrichtung der Veranstaltungen im Rahmen der Lehrevaluation (Durchschnittliche Globalbewertung: 3,5 (Schulnoten); s=1,37) sowie in Semesterabschlussgesprächen. Im Rahmen der Semesterabschlussgespräche wurde darüber hinaus von den Studierenden die Relevanz der Fächer für die spätere ärztliche Tätigkeit als gering wahrgenommen, was sich mit den Ergebnissen der Studie von Behmann und Kollegen deckt [16]. Aufgrund dieser Situation wurde beschlossen, dass bestehende Curriculum umzustrukturieren.

\section{Fragestellungen}

Bezugnehmend auf die beschriebenen Problematiken kann statuiert werden, dass kaum Konzepte bestehen, wie den Studierenden Wissen über das eigene Gesundheitssystem vermittelt und die Wichtigkeit und Praxisrelevanz des Themas verdeutlicht werden kann. Der fallbasierte Ansatz und die darin beschriebenen Fehler sollen die Relevanz des Themas für die ärztliche Tätigkeit hervorheben.

Es ergeben sich die folgenden 2 Fragestellungen:

1) Ist ein Methodenmix aus „Lernen aus Fehlern“(LaF) anhand eines echten Patientenfalles und eines Game-Based-Learning-Ansatzes zur Vermittlung von Wissen im Fach GGG geeignet?

2) Wie schätzen die Studierenden ihr Interesse sowie die Relevanz des Themas nach dem Besuch der umstrukturierten Seminare ein?

\section{Methoden}

Es wurden 4 Seminare entwickelt und im Wintersemester 2013/14 (WiSe) implementiert, die begleitend zu den Vorlesungen spezifische, kliniknahe Aspekte des Gesundheitssystems vertiefen. Die behandelten Themen waren aufgrund ihrer großen Relevanz die Schnittstellenproblematik zwischen dem ambulanten und stationären Sektor, die Beantragung einer Anschlussheilbehandlung $(\mathrm{AHB})$, die Beantragung einer Pflegestufe und die Adipositasprävention. Durch eine Fallgeschichte werden die 4 Seminare inhaltlich miteinander verknüpft. Auf der Basis der didaktischen Methoden „Lernen aus Fehlern“ [19, 22], „fallbasiertes Lernen“ [23-25] und „Game-Based Learning“ [26] besteht der Unterricht aus den 3 didaktischen Elementen „Gruppenarbeit“ [27], „Lösung von Quizaufgaben“ [28] und „Präsentationen“ [29]. Die Studierenden des Jahrgangs wurden auf insgesamt 16 Seminargruppen mit einer durchschnittlichen Seminargröße von 20-25 Personen aufgeteilt.

Eines der 4 Seminare basiert auf einem echten Patientenfall. An der Universität Witten/Herdecke wurde von den Autoren bereits erfolgreich ein fallbasiertes Kurskonzept mit der modifizierten Fallmethode nach Barrows [25] entwickelt, eingesetzt und evaluiert. Dabei kam es zu guten Ergebnisses bezüglich der Zufriedenheit, des selbsteingeschätzten Lernerfolgs und zur Erhöhung der Motivation sich mit dem Thema zu beschäftigen [30]. Dieses Kurskonzept diente als Vorlage für das Seminar an der LMU München, welches von der Arbeitsgruppe Gesundheit des Instituts für Didaktik und Ausbildungsforschung in der Medizin konzipiert wurde.

Die Autoren waren im Rahmen der Umstrukturierung für eins der 4 Seminare zuständig, welches hier eingehender beschrieben werden soll.
Im Rahmen des Seminars 2 wird der Weg eines krebskranken Patienten durch das Gesundheitswesen beschrieben (), der aufgrund mangelnder Absprachen und weiterer Schnittstellenproblematiken unbefriedigend behandelt wird. Der fallbasierte Ansatz und die darin beschriebenen Fehler sollen die Relevanz des Themas für die ärztliche Tätigkeit hervorheben [23].

Die Lernziele der Veranstaltung lauten wie folgt: Die Studierenden sind am Ende des Kurses in der Lage,

- ...die Schnittstellenproblematik zu erklären.

- ...die 3 Finanzierungsmodelle von Gesundheitssystemen zu benennen.

- ...zu erklären, in welchem Fall eine AU-Bescheinigung beantragt werden kann.

- ...den Prozess des AHB-Antrages zu beschreiben (wer stellt diesen und wo).

- ...die Rolle des Sozialdienstes beim Entlassmanagement zu erklären.

Im Seminar übernehmen die Studierenden die Rolle eines Hausarztes und analysieren in Gruppen mittels Factsheets die Probleme und erarbeiten Lösungsvorschläge.

Zu Beginn der Veranstaltung wird den Teilnehmern die Frage gestellt, warum Sie glauben, dass die Inhalte des Fachs GGG für sie interessant und wichtig seien und welche Relevanz das Themenspektrum für die spätere berufliche Arbeit haben könnte (Aktivierung der Teilnehmer nach Städli [31]). Nach einer kurzen Einführung in den Themenbereich „Internationaler Gesundheitssystemvergleich“ („Präsentationen“) wird der Fall ausgeteilt und von den Studierenden bearbeitet („Gruppenarbeit“). Nach der Fallgeschichte werden einzelne Aspekte zum Thema Schnittstellenproblematik, das Verfahren der Anschlussheilbehandlung (AHB), das Entlassmanagement und die Rolle des Sozialdienstes herausgearbeitet. Dabei nutzen die Studierenden Factsheets, die sie in Kleingruppen bearbeiten („Präsentationen“ und „Gruppenarbeit“). Die Ergebnisse werden in einem Quiz dargestellt („Lösung von Quizaufgaben“). Die Quizinhalte sind angelehnt an Fragen aus dem Staatsexamen. Von den Dozierenden werden die Themen des Quiz weiter vertieft („Präsentationen“). Zum Abschluss werden in der Gruppe mögliche Lösungsvorschläge für die Schnittstellenproblematik und der Ausgang des Falls diskutiert. Eine Übersicht des Kursablaufes ist in - Tab. 1 dargestellt.

Am Ende des Seminars evaluieren die Studierenden ihr Interesse am Thema (1 Item), verschiedene didaktische Aspekte des Seminars (10 Items), die Relevanz der Lehrinhalte in Bezug auf ihre spätere Tätigkeit als Ärztin/Arzt (1 Item), ihren Gesamteindruck zum Seminar ( 1 ltem) und ob sie an einem Pflichtwahlseminar zum Seminarthema (1 Item) teilnehmen würden. Der einseitige, maschinenlesbare Fragebogen (siehe Anhang, online) wurde im Wintersemester 2013/14 entwickelt und besteht aus 14 sechsstufig skalierten Items ( 1 = „Stimme voll zu“ bis 6 = „Stimme gar nicht zu“ bzw. 1 = Note 1 bis $6=$ Note 6 ) und 2 Freitextfragen (,Folgendes hat mir am Seminar besonders gut gefallen“ und „Folgendes sollte am Seminar unbedingt verbessert werden“). Auf Basis einer Faktorenanalyse erfolgte im Sommersemester 2015 eine Kürzung des Evaluationsbogens auf 9 geschlossene Items, die im Wesentlichen die gleichen Inhalte wie vorher abfragen (Fragebogen Version 2). Die didaktischen Aspekte wurden auf 5 Items gekürzt und die Abfrage 
- Tab. 1 Ablauf des Kurses „der einsame Patient“.

\begin{tabular}{|c|c|c|c|}
\hline Thema & $\begin{array}{l}\text { Dauer in } \\
\text { Minuten }\end{array}$ & Methode & Material \\
\hline Einleitung & 10 & Frontal & Kurzumfrage \\
\hline Input: Gesundheitssystemvergleich, Schnittstellenproblematik & 10 & Präsentation & Powerpoint \\
\hline Fallbesprechung & 15 & Gruppenarbeit, Diskussion & Fall, Tafel \\
\hline Quiz & 25 & Kleingruppenarbeit, Diskussion & Quiz, Factsheets \\
\hline Fall, Input: Sozialdienst, Entlassmanagement & 5 & Präsentation & Powerpoint \\
\hline Lösungen für Schnittstellenproblematik & 10 & Gruppenarbeit, Frontal & Tafel, Powerpoint \\
\hline Evaluation & 5 & Papierbasierte Evaluation & Fragebogen \\
\hline
\end{tabular}

zur Teilnahme an einem Pflichtwahlseminar wurde durch die Frage „Durch den Besuch der Veranstaltung wurde ich zu weiterem Selbststudium zum Thema „Gesundheitssystemvergleich“ motiviert“ ersetzt. Die beiden Freitextfragen wurden unverändert übernommen. Im Zuge der Fragebogenkürzung wurden zudem die sechsstufig skalierten Items in fünfstufig skalierte Fragen ( 1 = „trifft voll zu“ bis 5 „trifft gar nicht zu“ bzw. 1 = „sehr gut“, 2 = „gut“, 3 = „,befriedigend“, 4 = „ausreichend“, 5 = „mangelhaft“) geändert, um sie an die fakultären Evaluationsbögen anzupassen.

Des Weiteren wurden die Freitextfragen auf Häufigkeiten bestimmter Aussagen untersucht. Die häufigsten Aussagen, d. h. wenn mindestens 3 Nennungen vorhanden waren, wurden dabei thematisch zusammengefasst.

Die Kursstruktur und -inhalte wurden auf Basis der Evaluationsdaten und dem Feedback der Studierende gemäß dem Kern-Zyklus [32] regelmäßig angepasst.

Neben einer Schulung wurde den Dozierenden ein Studyguide und eine Powerpoint-Präsentation zur Verfügung gestellt. Bei dem interprofessionellen Team der Dozierenden, bestehend aus Ärztinnen, Pädagoginnen, Apothekerinnen und Gesundheits- und PflegewissenschaftlerInnen handelt es sich um Mitglieder der AG Gesundheit und Kommunikation des Instituts für Didaktik und Ausbildungsforschung in der Medizin, um weitere Mitarbeiterinnen und Mitarbeiter des Instituts und um Kolleginnen und Kollegen anderer Institutionen der medizinischen Fakultät.

Bezugnehmend auf die didaktische Methode Lernen aus Fehlern, basiert der Fall auf Fehler in der Behandlung von Kommunikation, die aufgrund der Schnittstellenproblematik entstanden sind. Die Besprechung des Falles, die Bearbeitung der dargestellten Aspekte und die gemeinsam erarbeiteten Lösungsstrategien orientieren sich dabei an den oben beschriebenen Grundvoraussetzungen für eine sinnvolle Bearbeitung von Fehlern im Lernkontext [19, 20].

\section{Ergebnisse}

Vom Wintersemester 2013/14 bis zum Wintersemester 2016/17 nahmen insgesamt 1804 Studierenden an dem GGG-Seminar teil. An der Evaluation nahmen 1162 teil, was eine Rücklaufquote von $64 \%$ ergibt. Nachfolgend werden die Evaluationsergebnisse zu den Kernthemen „Interesse am Thema“, „Einschätzung der fallbasierten Lehrmethode“, „Relevanz des Themas für den ärztlichen Alltag" und die Gesamtbewertung berichtet. Da zwei Fragebogenversionen und somit 2 Erhebungszeiträume (Wintersemester 2013/14 bis Wintersemester 2014/15 = $t_{1}$ und Sommersemester 2015 bis
Wintersemester 2016/17 = $t_{2}$ ) vorliegen, werden je Item ein Mittelwert bzw. eine Standardabweichung für $t_{1}\left(n_{1}=496\right)$ und für $t_{2}$ $\left(n_{2}=666\right)$ berechnet. Die Analysen der 4 ausgewählten Items zeigen, dass die Studierenden bei beiden Erhebungszeiträumen ihr Interesse am Seminarthema mit einem Mittelwert von 2,7 ( $s_{1}=1,3$; $\left.n_{1}=487\right)$ bzw. $2,3\left(s_{2}=1 ; n_{2}=665\right)$ eher im mittleren Bereich der Skala einordneten. Dennoch empfanden die Studierenden die Methode des fallbasierten Lernens als gut $\left(\mu_{1}=2,1 ; s_{1}=1,2 ; n_{1}=491\right.$ bzw. $\mu_{2}=1,8 ; s_{2}=0,8 ; n_{2}=663$ ) und schätzten die Relevanz des Themas als hoch ein $\left(\mu_{1}=2,5 ; s_{1}=1,6 ; n_{1}=482\right.$ bzw. $\mu_{2}=1,7 ; s_{2}=0,9$; $n_{2}=662$ ). Die beiden nachfolgenden Graphiken veranschaulichen mithilfe von 95-Konfidenzintervallen die beschriebenen Evaluationsergebnisse ( $\triangleright$ Abb. 1, 2).

Insgesamt bewerteten die Studierenden das Seminar auf einer sechs- bzw. fünfstufigen Notenskala durchschnittlich mit 2,1 ( $s_{1}=1$; $\left.\mathrm{n}_{1}=486\right)$ bzw. 1,9 ( $\left.\mathrm{s}_{2}=0,8 ; \mathrm{n}_{2}=659\right)$.

Bei den beiden Freitextfragen wurden die folgenden Aspekte am häufigsten genannt:

Frage 1: Folgendes hat mir am Seminar besonders gut gefallen: - Gute Struktur

- Patientenfall bzw. praktischer Bezug

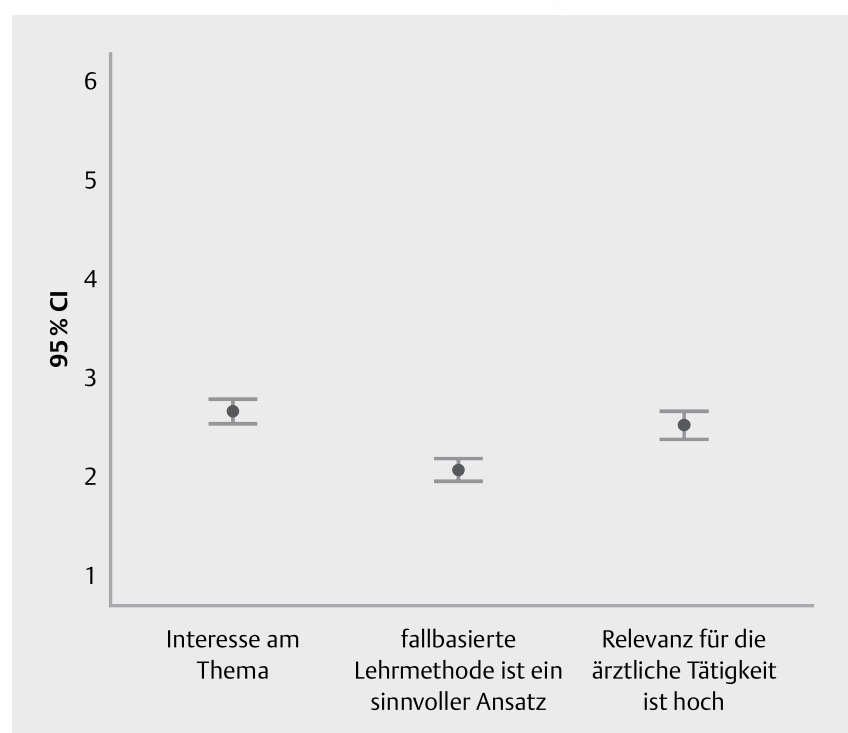

Abb. 1 Mittelwerte und 95\%-Konfidenzintervalle auf Basis der 6-stufigen Skala ( 1 = „Stimme voll zu“ bis 6 = „Stimme gar nicht zu“) am Erhebungszeitpunt $t_{1}$ (Wintersemester 2013/14 bis Wintersemester 2014/15). 


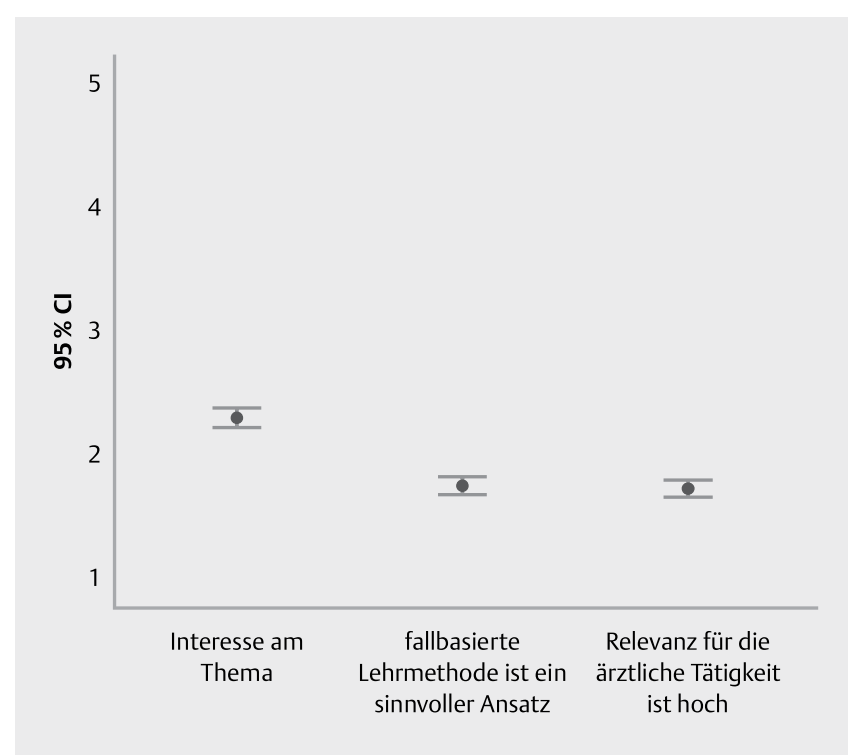

Abb. 2 Mittelwerte und 95\%-Konfidenzintervalle auf Basis der 5-stufigen Skala ( 1 = „trifft voll zu“ bis 5 = „trifft gar nicht zu“) am Erhebungszeitpunt $\mathrm{t}_{2}$ (Sommersemester 2015 bis Wintersemester 2016/17).

- Interaktivität

- Quiz

- Darstellung der verschiedenen Institutionen/Versicherungen im deutschen Gesundheitssystem

Frage 2: Folgendes sollte am Seminar unbedingt verbessert werden:

- Factsheets besser auf das Quiz abstimmen

- Keine Gruppenarbeit

\section{Diskussion}

Anhand der Evaluationsdaten kann festgestellt werden, dass die Methode „Lernen aus Fehlern “ anhand eines echten Patientenfalles geeignet ist, um Wissen im Fach GGG zu vermitteln, die klinische Relevanz des Faches zu verdeutlichen und das Interesse der Studierenden am Gesundheitssystem zu erhöhen. Damit widerlegen unsere Daten die Studie von Behmann und Kollegen [16], in der Medizinstudierende ein generell geringes Interesse am Thema Gesundheitssysteme angegeben haben und sie die Relevanz des Themas für ihre zukünftige berufliche Tätigkeit als gering einstuften.

Offenbar hat der Methodenmix des Seminars aus „Lernen aus Fehlern" anhand eines echten Patientenfalles und das Game-BasedLearning die geringe Motivation der Studierenden, sich mit dem Thema Gesundheitssysteme zu beschäftigen, erhöht. Zusätzlich scheint den Studierenden durch die Bearbeitung des echten Patientenfalles die hohe klinische Relevanz des Seminars deutlich geworden zu sein. Die allgemein hohe Akzeptanz der Seminarmethode durch die Studierenden zeigt, dass diese Art der Kursgestaltung geeignet scheint, die aus Sicht der Studierenden eher als uninteressant und zu theoretisch wahrgenommenen Themen zu vermitteln.

Auch die Beobachtungen der Dozierenden bestätigten das anfänglich geringe Interesse der Studierenden an der Thematik. Die
Aktivität der Studierenden nahm erst mit dem Einsatz des Falles merklich zu und erreichte seinen Höhepunkt bei der Beantwortung der Quizfragen. Zudem konnte, wie bereits in einer Arbeit von Städeli, beobachtet werden, dass eine Aktivierung der Studierenden zu Beginn des Seminars hilfreich ist, die Aktivität der Studierenden im gesamten Kursverlauf zu erhöhen [31].

\section{Limitationen}

Die Evaluation des vorliegenden Projekts basiert auf der Selbsteinschätzung der Studierenden und gibt daher keine Auskunft über den tatsächlichen Lernerfolg. Jedoch kann bei Berücksichtigung des Konzepts der Selbstwirksamkeitserwartung vermutet werden, dass Studierende, die sich kompetent fühlen auch tatsächlich relativ kompetent sind [33]. Da die Themen GGG nur einen kleinen Teil der Klausur einnehmen, können keine relevanten Aussagen zum Lernerfolg der Studierenden durch die Teilnahme am umstrukturierten Kurskonzept getroffen werden. Im Zuge der Restrukturierung der Themenfelder GGG und P\&G wurde außerdem auch die Klausur zu Beginn des neuen Kurskonzeptes neu konzipiert. Daher ist ein Vergleich mit den vorherigen Kursangeboten der LMU nicht möglich. Zudem gibt es keine Vergleichsgruppe, da das Kurskonzept flächendeckend für alle Studierenden der Medizin an der LMU angeboten wurde.

\section{FAZIT}

Betrachtet man die in der Arbeit von Behmann et al. festgestellte gering wahrgenommene Relevanz des Studienfaches seitens der Studierenden [16] mit den Ergebnissen dieser Studie, scheint das Kurskonzept, aus einem Methodenmix aus "Lernen aus Fehlern“ anhand eines echten Patientenfalles und Game-Based-Learning geeignet, um Wissen im Fach GGG zu vermitteln und die Relevanz für die spätere berufliche Tätigkeit zu verdeutlichen.

Die spätere Relevanz des Themenkomplexes GGG für die berufliche Tätigkeit wurde von den Studierenden scheinbar erkannt. Die geringe Motivation der Studierenden, sich mit der Thematik zu beschäftigen, konnte scheinbar reduziert werden, was auch durch die rückgemeldeten Beobachtungen der Dozierenden bestätigt wurde. Ein niedriges Interesse an der Thematik steht der höheren Einschätzung der Relevanz für die spätere klinische Tätigkeit gegenüber.

\section{Ausblick}

Das beschriebene Kurskonzept soll ab dem Sommersemester 2018 weiter entwickelt und auf die Inverted-Classroom-Methode umgestellt werden [34]. Zu diesem Zweck wird das Projekt durch „Lehre@ LMU“ gefördert. Ziel ist es, die Faktenvermittlung in eine OnlineSelbstlernphase zu verschieben, um in der Präsenzphase das erlernte Wissen anwenden und vertiefen zu können [35]. Zusätzlich wird die Patientengeschichte durch ein Video mit einem Schauspielpatienten ersetzt.

Bei weiteren Studien in diesem Feld wäre eine strukturierte Beobachtung der Studierenden im Unterricht hilfreich, um die direk- 
te Reaktion der Studierenden auf die einzelnen didaktischen Methoden differenziert zu erfassen.

\section{Literatur}

[1] Finkel ML, Fein O. Teaching medical students about different health care systems: an international exchange program. Acad Med J Assoc Am Med Coll 2006; 81: 388-390

[2] Prober CG, Khan S. Medical education reimagined: A call to action. Acad Med 2013; 88: 1407-1410

[3] Rowson M, Hughes R, Smith A et al. Global health and medical education - definitions, rationale and practice. Unpubl Work

[4] Xu K, Soucat A, Kutzin J et al. New perspectives on global health spending for universal health coverage. Geneva: WHO; 2018

[5] Mehta NB, Hull AL, Young JB et al. Just imagine: new paradigms for medical education. Acad Med 2013; 88: 1418-1423

[6] Frenk ], Chen L, Bhutta ZA et al. Health professionals for a new century: Transforming education to strengthen health systems in an interdependent world. Lancet 2010; 376: 1923-1958

[7] Gonzalo JD, Haidet P, Papp KK et al. Educating for the $21^{\text {st }}$-century health care system: An interdependent framework of basic, clinical, and systems sciences. Acad Med 2017; 92: 35-39

[8] Grüters-Kieslich A. Educating a new generation of physicians with awareness for global health and social responsibility. In: World Health Summit Yearbook 2013. Berlin: Charité - Universitätsmedizin Berlin; 2013: $18-19$

[9] Prideaux D. ABC of learning and teaching in medicine. Curriculum design. BMJ 2003; 326: 268-270

[10] Schneider N. Szenarien einer zukünftigen Gesundheitsversorgung aus Sicht der Versorgungsforschung. In: Robert Bosch Stiftung (Hrsg.). Ausbildung für die Gesundheitsversorgung von morgen. Stuttgart: Schattauer; 2011

[11] Chen L, Evans D, Evans T et al. The world health report 2006: working together for health. Geneva: World Health Organization, 2006

[12] Bligh J, Prideaux D, Parsell G. PRISMS: New educational strategies for medical education. Med Educ 2001; 35: 520-521

[13] Bodenheimer TS, Grumbach K. Understanding health policy: A clinical approach. McGraw Hill Book Co; 2008

[14] Anell A, Willis M. International comparison of health care systems using resource profiles. Bull World Health Organ 2000; 78: 770-778

[15] Simon M. Das Gesundheitssystem in Deutschland. Eine Einführung in Struktur und Funktionsweise. Huber; Bern: 2009

[16] Behmann M, Brandes I, Walter U. Die Lehre im Querschnittsbereich "Gesundheitsökonomie, Gesundheitssystem, Öffentliche Gesundheitspflege" an den medizinischen Fakultäten in Deutschland. Gesundheitswesen 2012; 74: 435-441

[17] Jacke CO, Frech J, Eikmann T et al. Was Medizinstudenten vom Gesundheitswesen wissen. Gesundh Ökon Qual Manag 2012; 17: 239-245

[18] Patel MS, Lypson ML, Davis MM. Medical student perceptions of education in health care systems. Acad Med 2009; 84: 1301-1306
[19] Oser F, Hascher T, Spychiger M. Lernen aus Fehlern Zur Psychologie des „negativen“ Wissens. In Althof W, (Hrsg.). Fehlerwelten: Vom Fehlermachen und Lernen aus Fehlern. Beiträge und Nachträge zu einem interdisziplinären Symposium aus Anlaß des 60. Geburtstags von Fritz Oser. Wiesbaden: VS Verlag für Sozialwissenschaften; 1999: $11-41$

[20] Kopp V, Stark R, Fischer MR. Förderung von Diagnosekompetenz in der medizinischen Ausbildung durch Implementation eines Ansatzes zum fallbasierten Lernen aus Lösungsbeispielen. GMS Z Für Med Ausbild 2007; 24 Doc 107

[21] Oser F. Aus Fehlern lernen. Wie „negatives Wissen“ hilft, es besser zu machen. Richt Falsch Von Fehlern Ihrer Richtigkeit 2009; 1: 4-6

[22] Stark R, Kopp V, Fischer MR. Case-based learning with worked examples in complex domains: Two experimental studies in undergraduate medical education. Learn Instr 2011; 21: 22-33

[23] Bowe CM, Voss J, Thomas Aretz H. Case method teaching: an effective approach to integrate the basic and clinical sciences in the preclinical medical curriculum. Med Teach 2009; 31: 834-841

[24] Thistlethwaite JE, Davies D, Ekeocha $S$ et al. The effectiveness of case-based learning in health professional education. A BEME systematic review: BEME Guide No. 23. Med Teach 2012; 34: 421-444

[25] Barrows HS. Problem-based learning in medicine and beyond: A brief overview. New Dir Teach Learn 1996 1996; 3-12

[26] Prensky M. Digital Game-Based Learning. Paragon House. St. Paul; Paragon House: 2007

[27] Haag L. Kooperatives lernen - kein Problem: effektive Methoden der Partner- und Gruppenarbeit (für Schule und Erwachsenenbildung). Klett-Schulbuchverl; Leipzig: 2004

[28] Reich K. Methodenpool. Im Internet: http://methodenpool.uni-koeln.de

[29] Meyer H. Praxisbuch Meyer: Unterrichts-Methoden. Berlin: Cornelsen; 1987

[30] Tolks D. Vergleichende Gesundheitssystemanalyse anhand von Patientenfällen: Evaluation eines studentischen internationalen Austauschprogramms und die daraus resultierende Curriculumsentwicklung. 2017; Dissertation.

[31] Städeli C, Grassi A, Rhiner K et al. Kompetenzorientiert unterrichten: das AVIVA-Modell. 2. Aufl. Bern: HEP; 2010

[32] Kern DE, Thomas PA, Hughes MT. (Hrsg.). Curriculum development for medical education: A six-step approach. 2. Aufl. Baltimore: John Hopkins University Press; 2009

[33] Epstein N, Fischer MR. Academic career intentions in the life sciences: Can research self-efficacy beliefs explain low numbers of aspiring physician and female scientists? PLOS ONE 2017; 12. Im Internet: http://journals. plos.org/plosone/article?id $=10.1371 /$ journal. pone. 0184543

[34] Lage M], Glenn JP, Treglia M. Inverting the classroom: a gateway to creating an inclusive learning environment. J Econ Educ 2000; 31: 30-43

[35] Tolks D, Schäfer C, Raupach T et al. An introduction to the inverted/ flipped classroom model in education and advanced training in medicine and in the healthcare professions. GMS J Med Educ 2016; 33: Doc46 
Seite 1 von 1

Wintersemester 2013/14

\section{„Evaluation Seminar 2: Einsamer Patient“}

Liebe Teilnehmerin, lieber Teilnehmer,

dieser Bogen wird maschinell ausgewertet. Markieren Sie eine Antwort bitte in der folgenden Weise: $0 \otimes 0$

Wenn Sie eine Antwort korrigieren möchten, füllen Sie bitte den falsch markierten Kreis und noch etwas darüber hinaus aus, ungefähr so: $O \otimes$

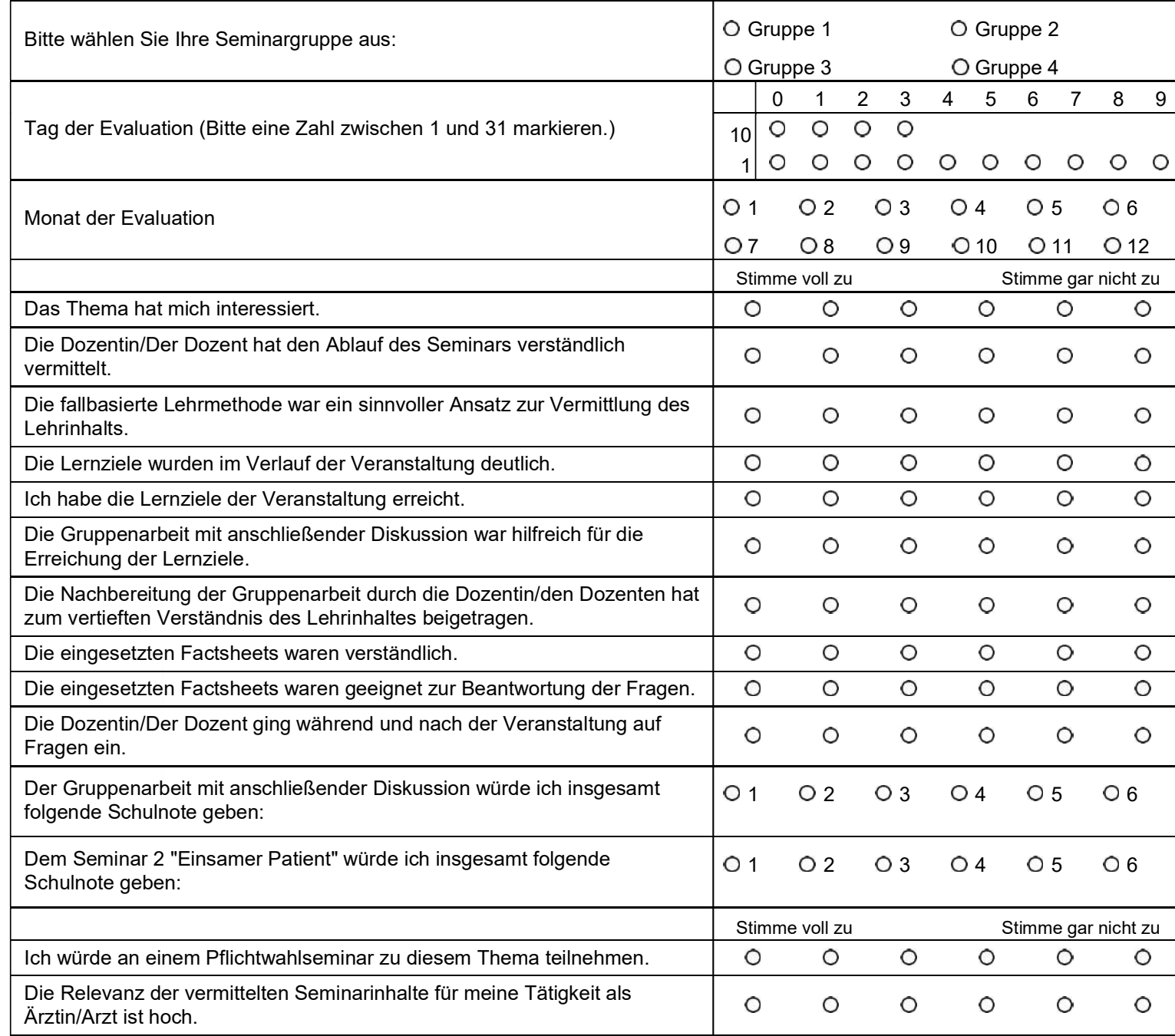

Folgendes hat mir am Seminar "Einsamer Patient" besonders gut gefallen: 